Pedagogía y Saberes n. ${ }^{\circ} 55$

Universidad Pedagógica Nacional

Facultad de Educación. 2021. pp. 105-120

\title{
Reformas educativas y reconfiguración de la subjetividad docente en Argentina
}

Educational Reforms and the Reconfiguration of Teacher's Subjectivity in Argentina Reformas educacionais e reconfiguração da subjetividade dos professores na Argentina

Cecilia Tosoni*

Tosoni, C. (2021). Reformas educativas y reconfiguración de la subjetividad docente en Argentina. Pedagogía y Saberes, (55). https://doi.org/10.17227/pys.num55-11382

* Profesora e investigadora en la Facultad de Educación Universidad Nacional de Cuyo (Argentina). Correo electrónico: ceciliatosoni@fed.uncu.edu.ar. Orcid: https://orcid.org/0000-0001-5433-5664 


\title{
Resumen
}

El artículo presenta una investigación sobre la tarea y la comprensión que de ella y de sí tienen los docentes desde la perspectiva de los estudios de gubernamentalidad. El estudio de caso se llevó adelante durante 2017-2018, a partir de entrevistas en profundidad realizadas a maestras de una escuela primaria de sectores populares del Gran Mendoza, Argentina. Particularmente, se indagó sobre la puesta en práctica de las reformas en la evaluación de los aprendizajes escolares introducidas por las reglamentaciones derivadas de la Ley de Educación Nacional № 26.206. En este trabajo, se analizan los dichos de las docentes, considerando que la normativa implica la ruptura del examen como forma de evaluación propia del disciplinamiento e introduce el ejercicio de la gubernamentalidad a través de la evaluación formativa, con características propias del monitoreo. Los resultados muestran cómo, en la puesta en práctica de las reformas, las maestras modifican su modo de evaluar y se resubjetivan como tales. Lo cual evidencia cómo se introduciría, a partir de la reforma de la evaluación de los aprendizajes, una forma de conducción de la conducta de la población propia de la gubernamentalidad neoliberal, característica de las políticas educativas recientes en la Argentina. Asimismo, se advierten indicios de una contraconducta de las docentes, cercana a la mística, que permite reconocerlas como agentes activos de la puesta en práctica de las decisiones de las autoridades educativas.

\section{Palabras clave}

reforma educativa; política gubernamental; evaluación; subjetividad

\begin{abstract}
The article presents a research about teachers' tasks and the understanding they have about their work and themselves from a perspective of governmentality studies. This case study was carried out based on thorough in-depth interviews with Elementary-school teachers in a school from popular sectors of Gran Mendoza, Argentina (2017-18). Particularly, they were inquired about the implementation of the reforms on the evaluation of school learning introduced by the regulations derived from the National Education Law Ner. 26.206. In this research, teachers' testimonies are examined, considering that the regulation involves a breaking of the exam as a form of evaluation distinctive of the disciplinary method and introduces the exercise of governmentality through the formative assessment, with proper monitoring. The results show how, in the implementation of the reforms, these teachers change their evaluation methods, and thus change their own role from mere evaluators to monitors or assessors. Starting from implementing a reform of the learning evaluation, this reveals how a new way of leading people behavior would be introduced proper of neoliberal governmentality, typical of the recent educational policies in Argentina. Likewise, there are clear signs of a teachers' contrasting behavior, close to mysticism or disinterested commitment, which lets them stand out as active agents of the implementation of the decisions of educational authorities
\end{abstract}

\section{Keywords}

educational reform; government policy; evaluation; subjectivity

\section{Resumo}

0 artigo apresenta uma pesquisa sobre a tarefa e a compreensão que têm os professores de si mesmos desde a perspectiva dos Estudos de Governamentalidade. 0 estudo de caso realizou-se em 2017-18, partindo das entrevistas feitas às professoras duma escola de Ensino Fundamental de setores populares da periferia de Mendoza, Argentina. Pesquisou-se sobre a prática das mudanças na avaliação das aprendizagens introduzidas pelos regulamentos derivados da Lei de Educação Nacional № 26.206 . Neste trabalho, analisam-se os ditos das professoras, considerando que a normativa implica quebrar o exame como forma de avaliação do disciplinamento e introduz o exercício da governamentalidade através da avaliação formativa, com características próprias do monitoramento. Os resultados mostram como na implementação das reformas, as professoras mudam seu modo de avaliar e reconfiguram sua subjetividade. Isto evidencia como se introduz, a partir da reforma da avaliação das aprendizagens, uma forma de condução da conduta da população própria da governamentalidade neoliberal, característica das políticas educativas recientes na Argentina. Além disso, assinala-se indícios duma contraconduta das professoras próxima à mística, que permite reconhecê-las como agentes ativas na implementação das decisões das autoridades educativas.

\section{Palavras-chave}

reforma da educação; política governamental; avaliação; subjetividade 


\section{Introducción}

La revista Pedagogía y Saberes nos propone, en este número, reflexionar sobre la figura de Paulo Freire a 100 años de su natalicio. El gran pedagogo brasilero dedicó su vida a mostrar que la educación liberaba a las personas de la opresión; su propuesta nos interpela como educadores latinoamericanos. Enseñar a leer la realidad, a interpretar nuestra experiencia, a pronunciar nuestro mundo se convierten en un desafío permanente para académicos, políticos, religiosos, maestros, familias y organizaciones de la sociedad civil que buscan educar, no sin conflictos y contradicciones. Por otro lado, la difusión del pensamiento de Paulo Freire ha generado expectativas en torno a la posibilidad de una educación con carácter emancipador que contrasta con su institucionalización en sistemas educativos y su instrumentalización desde una perspectiva de aprovechamiento individual de títulos y acreditaciones. Asimismo, la acción pedagógica aparece atrapada en una trama de relaciones que convierte al maestro en agente estatal y a su propuesta enseñanza en la rutinización de saberes y prácticas impuestas desde programas generados por los funcionarios de los distintos gobiernos.

Esta situación ha llevado, en algunas oportunidades, a pensar en una educación liberadora a puertas cerradas. El maestro o la maestra en su aula lleva adelante una acción educativa emancipadora, en la medida en que niños y niñas, adolescentes o adultos aprenden a reflexionar la propia experiencia a partir de sus propuestas de enseñanza aprendizaje innovadoras y democráticas. Sin embargo, esta perspectiva voluntarista, si se quiere, centrada en la decisión ética del docente diluye, entiendo, su carácter emancipador. Pensar una acción educativa emancipadora, al margen de la trama de relaciones en las que se encuentra el maestro, descontextualiza la tarea y la despolitiza. La descontextualiza porque el maestro enseña a grupos particulares que pertenecen a distintos sectores sociales, con historias y experiencias disímiles. Y la despolitiza, porque ignora sus condiciones de trabajador, minimiza el control las autoridades educativas sobre él y su labor. Entiendo, que debemos asumir una mirada política sobre la educación e indagar la trama de relaciones de poder en las que los docentes desarrollan su tarea para comprender su complejidad, particularmente, la de quienes enseñan en instituciones a la que asisten los niños y niñas de sectores populares.

Recuperar una mirada política sobre la educación, en general, y sobre la acción del docente en particular, se hace necesaria para reconocer que las decisiones educativas de los gobiernos no son neutrales, sino que pretenden dirigir la conducta de los ciudadanos a través de ellas. Esto nos lleva a preguntarnos ¿en qué medida las acciones de los docentes son conducidas por el Estado a través de sus políticas educativas?, ¿pueden los maestros tomar distancia de aquello que pretenden los distintos gobiernos de su tarea y asumir una postura propia? y ipueden actuar a contrapelo de lo pautado por las autoridades educativas?

En este sentido, entiendo que la perspectiva de los estudios de gubernamentalidad, desarrollada a partir de las categorías propuestas por Michel Foucault, posibilita comprender la educación fuera del triángulo docente-saber-estudiante, para dar cuenta de la trama de relaciones de poder en la se encuentran los distintos agentes educativos. Considerar la complejidad de la educación como forma de intervención del Estado en la sociedad, reconocer que no hay un alguien -el maestro-, que libera a otro - el alumno-e indagar sobre cómo los gobiernos buscan direccionar la vida de las poblaciones da lugar a una comprensión más modesta y realista de la tarea educativa (Noguera, et ál., 2013).

A continuación, presento una parte de los resultados de la investigación ${ }^{1}$ que llevé adelante en 2017 2018, cuyo objetivo fue comprender los cambios en la evaluación escolar a partir de la identificación de relaciones de poder entre docentes, niños, padres directivos y supervisora en el marco de las reglamentaciones vigentes derivadas de la Ley de Educación Nacional N² 26206 (2006) en la Provincia de Mendoza (Argentina). ${ }^{2}$ El estudio abarcó la identificación de las fundamentaciones sobre las reformas en la evaluación

1 Este artículo contiene parte de los resultados de mi tesis de Doctorado: Evaluación escolar, conflictos y respuestas. Un estudio sobre los discursos y las prácticas de los diferentes agentes educativos referidos a la evaluación de los aprendizajes en una escuela de sectores populares, dirigida por la Dra. Norma Fóscolo, codirectora Dra. Romina Elisondo, Facultad de Ciencias Humanas, UNRío Cuarto (Córdoba, Argentina)

2 En Argentina, las decisiones políticas educativas tienen dos instancias, una nacional en la que se establecen directrices generales (Ministerio de Educación de la Nación) y otra provincial, en la que estas se precisan en reglamentaciones para dicha jurisdicción (Ministerios provinciales, direcciones o consejos provinciales). En Mendoza la Dirección General de Escuelas (DGE). La Ley de Educación Nacional (2006) asumió como principio la inclusión educativa frente a situaciones de exclusión social que viven grandes sectores de la población. En 2012 se aprobó en el Consejo Federal de Educación (integrado por los ministros de cada provincia) el Acuerdo Federal 174/12 que contiene pautas para la reforma de la evaluación de los aprendizajes atendiendo al principio mencionado. La Provincia de Mendoza, llevó adelante estos cambios a partir de la Resolución N 681712 DGE sobre el Régimen Académico para el nivel primario de la educación. En 2016 con el cambio de gestión fue apenas modificada por la Resolución N¹155/16 DGE 
de los aprendizajes presentes en discursos académicos y oficiales, luego, un análisis sociolingüístico de las reglamentaciones que precisaron las modificaciones en las prácticas escolares. Por último, un estudio de caso, para conocer el significado que los propios agentes educativos dan a la evaluación y los conflictos emergentes en el día a día escolar. Para lo cual llevé adelante un trabajo de campo de tipo etnográfico en una escuela de sectores populares; indagué, entonces, en qué medida su puesta en práctica de las reformas en la evaluación impacta en la comprensión que las maestras tienen de su tarea y de sí mismas mediante entrevistas en profundidad.

Con este artículo, busco contribuir a la discusión sobre la tarea docente atendiendo a la complejidad política que supone. Es decir, dar cuenta de la trama de relaciones de poder en la que se encuentran los maestros, particularmente, quienes trabajan en escuelas de sectores populares; de las formas de sujeción promovidas por las políticas educativas a través de distintas prácticas escolares y de sus respuestas frente a ellas en un caso particular. El estudio realizado permite reconocer continuidades en la forma de direccionamiento de los ciudadanos a través de la educación por parte del Estado, pese a las diferencias promocionadas mediáticamente por los funcionarios de las distintas gestiones de gobierno. ${ }^{3}$ Lejos de los moralismos que promueven ser un educador emancipador ignorando los contextos particulares $\mathrm{y}$, distantes de posiciones que suponen al docente como simple ejecutor de las decisiones de estatales, este trabajo aporta una reflexión sobre cómo los maestros lidian con las políticas educativas y reconfiguran su subjetividad a partir de sus propios relatos (Beech et ál., 2016).

3 Durante los años 2017-2018, Mauricio Macri estaba a cargo del gobierno nacional y Alfredo Cornejo era gobernador de la Provincia de Mendoza, ambos formaban parte del Frente Cambiemos (orientación liberal republicana) que derrotó al Frente para la Victoria (integrado por el Partido Justicialista y otros de izquierda (orientación nacional y popular) en las elecciones de 2015. Desde 2003, este frente gobernó el país, primeramente, con Néstor Kirchner (2003-2007) y luego, en dos periodos consecutivos con Cristina Fernández de Kirchner (2007-2011 y 2011-2015). Durante el gobierno de Cristina Fernández de Kirchner la provincia de Mendoza estuvo gobernada por Celso Jaque y Francisco Pérez, quien también pertenecían al Frente para la Victoria. Mediáticamente, sobre todo en las elecciones 2015, la política argentina se presentó atravesada por una 'grieta' entre quienes apoyaban al Frente Cambiemos con propuestas neoliberales explícitas (sectores de clases medias y altas) y el Frente para la Victoria con un programa anclado en reforzar las reivindicaciones sociales de los grupos postergados (sectores medios y populares).

\section{Herramientas teóricas para pensar lo que pasa en las escuelas}

En el marco de los estudios de gubernamentalidad, recupero algunas categorías foucaultianas que nos permiten comprender las formas escolares de socialización, en las que se enlazan relaciones de poder ${ }^{4} \mathrm{y}$ saber con las formas de configuración de las subjetividades; asimismo, posibilitan vincular lo que acontece en las instituciones con las decisiones políticas de los gobiernos (Veiga-Neto, etál., 2011). Este utilitarismo metodológico pretende que sus conceptos se conviertan en los lentes para mirar nuestro presente, a fin de reconocer en qué medida la educación como política de Estado se convierte en una forma de conducción de la conducta de las poblaciones y de qué manera modela a los sujetos que participan en ella, particularmente, a los docentes de escuelas de sectores populares (Veiga-Neto, 2013, p. 10).

Para ello, tomo como punto de partida el concepto de disciplinamiento desarrollado por Michel Foucault (Foucault, 1989). Utilizado por las pedagogías críticas para dar cuenta de las relaciones de poder en las escuelas. El disciplinamiento como forma de relación de poder ha sido visto como opresivo, sin embargo, como señala Julia Varela, es importante reconocer su carácter productivo, es decir, modelador de las experiencias de sí mismo, en las que se gesta la subjetividad de los individuos (Varela, 2001). En segundo lugar, introduzco el concepto de gubernamentalidad a fin de dar cuenta de una nueva modalidad en el ejercicio del poder propia de la contemporaneidad. El concepto de gubernamentalidad, desarrollado por Foucault, refiere al arte de gobernar y la racionalidad que le es propia, surgida en occidente a partir de la pastorado cristiano (Foucault, 2006). En tercer lugar, me detengo en las características de las políticas educativas recientes y en las modificaciones evaluación de los aprendizajes que han implicado. En cuarto lugar, presento el contexto educativo argentino, en el que se han implementado reformas a partir de la LEN № 26.206, cuyo eje es la inclusión educativa. Al final de este apartado desarrollo las preguntas que guiaron específicamente mi investigación sobre las

\footnotetext{
4 Para Michel Foucault el poder no es una cosa ni tampoco es sinónimo de violencia. El poder existe en cuanto relación que se ejerce, implica una acción sobre otras acciones y supone la estructuración de un campo (Foucault, 2001, p. 255). Las relaciones de poder se diferencian de las de dominación y explotación, porque constituyen el modo en cómo cada uno se vincula consigo mismo y con los otros. Las relaciones de poder son móviles y adoptan distintas formas, son productivas, es decir, incitan, inducen, facilitan o dificultan acciones, para producir determinados resultados, particularmente, diferentes modos de ser sujetos (Varela, 2001).
} 
modificaciones en las prácticas de evaluación de los aprendizajes y el modo en que las maestras se reconocen como tales a partir de ellos.

\section{La escuela y el disciplinamiento}

El disciplinamiento como modalidad de ejercicio del poder surge en Europa a partir del siglo XVII, en la que aparecen diferentes instituciones cerradas. El hospital, la escuela, el campamento militar, la cárcel y la fábrica configuran espacios de disciplinamiento donde quienes asisten se ven coaccionados a actuar siguiendo determinadas pautas (Foucault, 1989). Es un poder modesto, suspicaz, calculado, permanente que se hace efectivo en instituciones cerradas a partir de técnicas precisas: la inspección jerárquica, la sanción normalizadora y el examen (Foucault, 1989). La vigilancia jerarquizada implica que cada uno de los observantes es observado, es decir, que un mecanismo relacional coloca a uno como observador del otro. La sanción normalizadora, supone una corrección por repetición que garantizará la adquisición de desempeños específicos. El examen es un instrumento privilegiado de disciplinamiento, ya que combina la inspección jerárquica con la sanción normalizadora. El examen, como procedimiento, contribuye a normalizar conductas, a regularizarlas dentro de un grupo particular, provocando docilidad y también posibilitando el mejoramiento de los desempeños, registrados y permanentemente jerarquizados.

En la escuela, el examen coloca al docente en una relación de poder/saber con el alumno. El maestro califica sus saberes y, también, produce un conocimiento sobre él (Foucault, 1989). Así, además de identificar los conocimientos aprendidos, obtiene una información sobre el sujeto que se encuentra a su disposición para ser comparado, diferenciado, jerarquizado, gratificado o sancionado y, en última instancia, excluido del grupo clase, cuando no promociona. La ceremonia del examen implica un acto de visibilización del evaluado, una recopilación de información que lo convierte en un caso a comparar con un modelo preestablecido. El examen concluye con una jerarquización que excluye a quienes no responden al modelo establecido (Foucault, 1989).

\section{Gubernamentalidad como forma de ejercicio del poder}

Esta nueva manera de ejercer el poder, la gubernamentalidad, implica un ejercicio autolimitado del poder. Un gobierno frugal caracterizado por el cálculo estratégico de intervenciones por parte de aquel que pretende gobernar a los otros. Esta nueva racionalidad consiste en el ejercicio de un gobierno mínimo, autorregulado
(Foucault, 2007). No es un ejercicio del poder que sea limitado externamente, o que deba ser más o menos legitimado por un pacto para actuar. Se trata de un ejercicio que se limita según lo que conviene o no hacer. Así, el ejercicio de la ratio gubernamental consiste en una práctica y su racionalización dirigida a una intervención mínima de la autoridad, pero exitosa en términos de alcanzar los efectos deseados.

La gubernamentalidad, como forma de encaminar la conducta de los hombres, instala un régimen de veridicción, una alianza entre saber y poder, una grilla de inteligibilidad de la acción humana desde el cálculo de intereses y la irreductibilidad individual de la decisión. Se trata, pues, de considerar al otro y a sí mismo como individuo, capaz de decidir por sí lo que le conviene o no hacer. "Es una persona cualquiera o, en fin, se lo trata como a cualquier otra persona que invierte en una acción, espera de ella una ganancia y acepta el riesgo de una pérdida" (Foucault, 2007, p. 292). Quien gobierna manipula intereses, identifica riesgos y mide hasta qué punto son controlables.

La gubernamentalidad es, entonces, una forma particular de dirigir la conducta de los hombres, que extiende la racionalidad económica a la comprensión de otras actividades educación, trabajo, relaciones de pareja y que modula a los sujetos en cuenta sujetos de interés. Esta forma particular de comprender al individuo se desarrolla en occidente particularmente en los últimos decenios y fue promovida por el neoliberalismo norteamericano (Foucault, 2007).

El sociólogo inglés Nikolas Rose afirma que la gubernamentalidad es una forma particular de intervención del Estado en la sociedad, que comprende las deliberaciones, las estrategias los dispositivos ideados por las autoridades para conducir a los individuos, a través de sus propias elecciones (Rose, 2007). Esta modalidad enfatiza al individuo como agente activo, capaz de desarrollar habilidades que le permitan enfrentan los riesgos. Sus consecuencias serían una mutación profunda de las formas de pensar y actuar de los sujetos y nuevos modos de problematizar e intervenir el Estado en la sociedad. Estas nuevas formas de pensar, no son patrimonio de las autoridades de gobierno, sino que afectan los deseos y las aspiraciones de cada uno. Ya que promueven la autoinspección, la conciencia de uno mismo de la mano de un saber experto sobre la psiquis individual (Rose, 1990).

\section{Políticas educativas recientes, nuevos discursos, nuevas prácticas}

Stephen Ball señala que a partir de los años 80 se habrían implementado políticas vinculadas a la gestión empresarial en la educación instalando una 
nueva racionalidad (Ball, 2001). Es decir, una forma de inteligir la educación y de plantear sus problemas que daría lugar a la introducción de ejercicio del poder propio de la gubernamentalidad en el ámbito educativo. En los últimos treinta años, las políticas educativas se basan en el paradigma de Nueva Gestión Pública, que recupera conocimientos e instrumentos de gestión de empresas a la administración del Estado. En el ámbito de la administración escolar, los principios de la Nueva Gestión Pública se traducen en una mayor autonomía de los centros educativos, un enfoque gerencialista del director, rendición de cuentas a partir de la evaluación de los desempeños en función de los estándares establecidos, incentivos por los resultados y restructuración de la profesión docente (Verger et ál., 2015).

En esta línea, Carlos Noguera Ramírez y Dora Marín Díaz señalan que durante el siglo Xx se genera una forma de comprender la intervención educativa del Estado en la sociedad que responde al ejercicio de poder gubernamental neoliberal (Noguera etál., 2012). De la mano de las pedagogías activas, que pone el acento en el carácter protagónico del sujeto que se transforma en cuanto que aprende, se produce el desplazamiento a una sociedad del aprendizaje, en la que los gobiernos consideran que los individuos se forman permanentemente. Los nuevos discursos educativos buscan promover y garantizar espacios en los que cada individuo tenga condiciones para desarrollar sus propias habilidades, capacidades y competencias que le permitan adaptarse a un mundo con una economía en constante movimiento. "Un capital humano que se gerencia a sí mismo, que produce como tal” (p. 28).

Silvia Grinberg desde la perspectiva de la gubernamentalidad estudió las políticas educativas instauradas en la Argentina de los años 90. Abordó, concretamente, los discursos y las prácticas que se establecen los documentos oficiales del Ministerio de Educación de la Nación (Grinberg, 2008). La autora da cuenta de cómo se introdujeron nuevas reglas del discurso pedagógico desde los documentos del Ministerio de Educación de la Nación, que provocaron la irrupción del ejercicio del poder propio de la gubernamentalidad en las prácticas educativas a partir de las reformas iniciadas por la Ley Federal de Educación en la Argentina en 1996, pues promovieron la gestión como eje de los cambios, introduciendo el cálculo de los riesgos, procurando condiciones para la ampliación de la capacidad de decisión y acción individual. De manera que se invisibiliza el poder mientras crece la sensación de libertad.

Por otra parte, Grinberg plantea que, a partir de la década de los noventa, las prácticas de evaluación no responden a la lógica del disciplinamiento, siguiendo la modalidad del examen; sino que combinan lógicas del monitoreo e intervención (Grinberg, 2015). El monitoreo, implica el seguimiento continuo y una intervención anticipada y calculada en vistas a alcanzar mejores resultados. Supone un conjunto de prácticas de evaluación que entrenan al individuo para enfrentar dificultades, no solo se refieren a conocimientos, sino también a la dimensión afectiva de las personas. Esta nueva modalidad de evaluación es correlativa con un tipo de intervención que no sanciona, sino que simplemente orienta, detecta riesgos y promueve la autoevaluación suscitando una conducta, una llamada a revisarse, a aprender y mejorar de modo permanente. El monitoreo y su forma de intervención mínima se corresponde con la gubernamentalidad como forma de ejercicio del poder. Este nuevo modelo de evaluación procura una regulación individual. Los maestros tienen que hacer un seguimiento y proponer acciones diferenciadas, orientar, no sancionar. La evaluación pierde ese carácter para convertirse en una constante intervención diferenciada, que supone además la autoevaluación y la revisión de sí. Así, cada uno es conminado a involucrarse a tomar decisiones para reorientar su conducta: "de manera que, la evaluación y la medición tienen y producen efectos en tanto tecnologías que nos ponen a hacer algo con nosotros mismos" (p. 166).

\section{El contexto argentino}

En la Argentina del nuevo milenio, en un contexto de pauperización y creciente desigualdad social, las medidas gubernamentales pusieron el acento en la educación como respuesta política privilegiada. A partir de la Ley de Educación Nacional № 26.206 de 2006 (LEN), se reinstala la centralidad del Estado desde la expectativa de atender a las desigualdades sociales, particularmente desde la educación; adquiere importancia el concepto de inclusión educativa y el Estado reafirma su rol de garante del derecho a la educación (Gluz, 2016). La demanda de inclusión educativa de los sectores postergados tomó cuerpo en la necesidad de romper con estructuras propias de la escuela tradicional, consideradas como obstáculos para completar su escolaridad. En este sentido, la modificación de la evaluación escolar se convirtió en objetivo de las políticas educativas, particularmente, desde la LEN. Las normativas derivadas buscaron modificar la evaluación de los aprendizajes, a partir nuevos discursos sobre la evaluación y la promoción de otras prácticas docentes.

Atendiendo a la centralidad de la evaluación en la experiencia educativa de la vida de los individuos y su vinculación con Estado me pregunté: ¿En qué medida las reformas de la evaluación escolar introducen 
nuevas relaciones entre docentes, niños, directivos, padres y autoridades educativas?, ¿de qué manera estos cambios generan en los docentes otra forma de reconocerse como maestros? y ¿cómo estas relaciones muestran otra forma de intervención del Estado en la vida cotidiana de los individuos?

Desde esta perspectiva foucaultiana especifiqué mi indagación, entiendo que el tipo de evaluación tradicional se vincula con el modelo del examen, relacionado al ejercicio del poder disciplinario. Asimismo, considero que la evaluación formativa, propuesta en las reglamentaciones para reemplazarla, introduciría modificaciones en las relaciones entre maestros y niños, entre la escuela y los padres. Este tipo de evaluación es a fin al modelo del monitoreo, cuyas características son propias del ejercicio de la gubernamentalidad. Desde allí me pregunté: ¿Cómo se modifican las prácticas docentes a partir de las formas de evaluación propuestas en las reglamentaciones derivadas de la LEN?, ¿implican un reemplazo del examen, por el modelo del monitoreo?, ¿cómo los cambios en evaluación modifican el modo de comprensión de los docentes sobre su tarea y sobre sí mismos? y ¿en qué medida las nuevas prácticas y la comprensión que tienen de ellas y de sí las docentes responden a una nueva forma de intervención del Estado en la sociedad, propias de la gubernamentalidad neoliberal?

\section{Metodología}

Con el propósito de responder dichas preguntas realicé una investigación cualitativa de carácter exploratorio mediante un estudio etnográfico; la elección de un solo caso me demandó una descripción densa para proveer de base empírica fehaciente a la comprensión de la cotidianidad escolar desde la perspectiva sociológica elegida, que pone en foco las distintas formas de ejercicio del poder disciplinario y gubernamental (Marradi, 2010). Esta metodología es fructífera en la medida en que permite dar cuenta de la complejidad de los procesos políticos, identificar las relaciones que se establecen concretamente entre los distintos agentes y entender cómo interpretan sus acciones (Miranda, 2011). Asimismo, se vincula estrechamente con la perspectiva foucaultiana porque las técnicas utilizadas posibilitan la reconstrucción de las prácticas cotidianas y los discursos situados, permitiendo reconocer los saberes legitimadores, los modos de sujeción y formas de subjetivación propias de relaciones de poder desplegadas en ámbitos específicos. En el día a día escolar, los distintos agentes educativos son quienes hacen las políticas educativas que fueron escritas por las autoridades de gobierno y discutidas y fundamentadas por especialistas (Beech et ál., 2016). La puesta en marcha de las políticas supone una acción por parte de sus practicantes; así, no hay un proceso lineal de aplicación de las normas, estas son interpretadas por directivos, docentes, alumnos y padres que deben resolver sus tensiones, sus lagunas y efectos no previstos en el día a día escolar (Miranda, 2011). Por lo que es posible, a través de la metodología etnográfica, reconocer las resistencias y rechazos a las formas de conducción de los agentes educativos, en particular, de los docentes.

Específicamente, me propuse llevar adelante una etnografía crítica, a fin de recuperar la interpretación que hacen los que no son escuchados habitualmente en sus discursos situados y en sus tácticas específicas en los espacios locales (Miranda, 2011). Por ello busqué rescatar lo que dicen los maestros, cómo describen sus acciones, cómo argumentan sus decisiones y cómo se comprenden a sí mismos a partir de las modificaciones en la evaluación de los aprendizajes que implicaron las reglamentaciones derivadas de la Ley de Educación Nacional № 26.206.

Como señalé, dicha ley tiene como principio la inclusión educativa. Su objetivo es garantizar la permanencia de los niños en situación de vulnerabilidad en la escuela. Por ello, tomé como caso a estudiar una escuela primaria a la que asisten niños de sectores populares reconociendo su heterogeneidad ${ }^{5}$, a fin de observar qué situaciones, qué discursos y prácticas se generan en torno a la evaluación de los aprendizajes, cuando existe desigualdad entre los grupos más vulnerables dentro de la propia institución. Elegí una institución del distrito de Las Tortugas (Departamento de Godoy Cruz, Mendoza). La mayoría de los habitantes de esta zona pertenece a los sectores populares debido a que los barrios fueron construidos por el gobierno de la Provincia de Mendoza para familias de recursos insuficientes y erradicación de

\footnotetext{
5 Benza retoma la división tripartita de clases altas, medias y populares de los trabajos clásicos de la estructura social argentina, que consideran como "proxi" los lugares asignados por la división del trabajo, es decir, las posiciones u ocupaciones en la esfera laboral (Benza, 2016). Pablo Semán y Cecilia Ferraudi Curto señalan que los sectores populares, en la Argentina, han sido atravesados en las últimas décadas por dinámicas económicas y políticas que generan heterogeneidades y desigualdades entre sus distintos segmentos (Semán, et ál., 2016).
} 
villas inestables a partir de la década de $1970 .{ }^{6}$ En la selección del caso a estudiar, dos grupos de $7^{\circ} \stackrel{0}{ }$ año, consideré el nivel de ingresos de la familia tomando en cuenta la ocupación de los padres, el nivel de escolaridad de los padres y las madres y los barrios de procedencia de los niños. El grupo elegido se caracterizó por la presencia de clases populares con la participación de clases medias. Asimismo, el grupo de docentes entrevistadas era diferenciado en cuanto a su edad y experiencia.

Para conocer lo que ocurre en la escuela a propósito de la evaluación de los aprendizajes, desde un enfoque etnográfico consideré fundamental relevar datos en mi investigación mediante la observación participante y entrevistas (Yuni et ál., 2005). En el caso de las maestras, tomé en cuenta, especialmente, las entrevistas en profundidad que realicé a ocho docentes de $7^{\circ}$ año y a la maestra de apoyo de la institución (nueve en total). Indagué sobre la forma en que evalúan, el modo en como resuelven dificultades, si la práctica de las reglamentaciones vigentes ha provocado cambios en su actuación y su vinculación con autoridades y otros organismos del Estado. El hilo conductor de las guías para las entrevistas fue la distinción pedagógica entre evaluación tradicional y formativa, eje de las modificaciones en las reglamentaciones. Estos conceptos me permitieron establecer una comunicación que dio lugar a la explicitación de sus propias posiciones, lo que me facilitó la codificación posterior. Todas las entrevistas fueron grabadas. Posteriormente realicé su transcripción, obteniendo un documento por cada una de ellas. Esto me permitió su procesamiento por medio del programa Atlas ti, a partir del cual obtuve las matrices de datos en las que organicé a información obtenida.

6 En el distrito existen numerosos barrios edificados por el Instituto Provincial de la Vivienda (IPV), Gobierno de Mendoza, para atender las demandas de familias de bajos recursos que buscan acceder a una casa propia. Las distintas operatorias del IPV respondieron a grupos diferenciados, trabajadores municipales sin vivienda propia, erradicación villas de emergencias o que familias habían sido afectadas por el sismo de 1985, entre otros. Para los mendocinos algunos de estos barrios del sudeste de Godoy Cruz son sinónimo de pobreza, zona "roja" o "peligrosa." Los medios de comunicación los mencionan con frecuencia en las crónicas policiales, las cuales refieren a la guerra de bandas y a las víctimas de los tiroteos. En algunas oportunidades, son noticia las acciones solidarias de sus vecinos y aparecen en la sección de cultural o educativa. Los habitantes de algunos de estos barrios se ven obligados a declarar otro domicilio para conseguir trabajo, retirar los resúmenes de cuenta de los celulares en la sede del correo privado porque estos no llegan a sus casas. Incluso, en algunos casos, los servicios de emergencias médicas no ingresan mientras las ambulancias públicas lo hacen acompañadas por la policía.
Las anticipaciones de sentido que guiaron $\mathrm{mi}$ indagación fueron:

1. En las prácticas evaluativas de los docentes, el reemplazo de la evaluación tradicional por la evaluación formativa permite la introducción del modelo del monitoreo en tensión con el examen, generando la presencia de ambas formas de ejercicio de poder (disciplinamiento y gubernamentalidad).

2. El modo de subjetivación de los docentes se modifica atendiendo a los requerimientos del monitoreo como modo de evaluación propio de una relación de poder gubernamental.

\section{Resultados de la indagación}

Las reformas propuestas para la evaluación de los aprendizajes derivadas de la LEN № 26.206 se efectivizaron en la jurisdicción de la Provincia de Mendoza con la Resolución $\mathrm{N}^{\circ} 0681 / 12 \mathrm{DGE}^{7}$ que regula el Régimen Académico de nivel primario, junto con la reforma de los artículos del 22 a 38, establecida por la Resolución $N^{\circ} 1155$ DGE del 2016 . En ellas busqué el primer indicio de la introducción de relaciones propias de la gubernamentalidad en la escuela (Tosoni, 2019); en el análisis de sus textos encontré que promueven el reemplazo de la evaluación tradicional o de control, por una formativa, continua, atenta al proceso individual en función de los resultados. Lo cual implica una intervención anticipada por el docente, que además debe ayudar al alumno a enfrentar dificultades y a revisar su actuación para alcanzar los logros esperados. Este tipo de evaluación se vincula con el monitoreo en la medida en que sigue a los individuos en la particularidad del proceso que cada uno lleva, no sanciona, sino que acompaña a lograr los conocimientos esperados, tenidos como metas. La evaluación educativa propuesta está lejos de la clasificación de alumnos y de su posterior jerarquización, se enfoca en lo que cada uno realizó en función de sus diferencias y promueve la autoevaluación; el docente orienta, acompaña y comprende en su individualidad, y el modelo de evaluación propuesto responde al monitoreo siendo afín al ejercicio del poder como gubernamentalidad (Grinberg, 2015).

A partir de este primer indicio, me pregunté cómo los distintos agentes educativos se apropian de la forma de comprensión de la evaluación presente en los

7 DGE Dirección General de Escuelas, máximo organismo educativo de la Provincia de Mendoza, tiene rango de ministerio. Las resoluciones son el último eslabón de la cadena normativa. Tienen una alta exigibilidad por parte de las autoridades jurisdiccionales. 
documentos oficiales mencionados. Así, analicé los dichos de las docentes para conocer si los cambios provocados por la puesta en práctica de las nuevas reglamentaciones confirmaban la introducción de la gubernamentalidad como forma de ejercicio del poder, a través de la práctica de la evaluación formativa, cercana a monitoreo, por una parte. Y, por otra, si esto modificaba el modo de comprensión que ellas tienen de su tarea y de sí mismas, es decir, el modo en que se resubjetivan. A continuación, expongo cómo las docentes comprenden y llevan adelante las prácticas de evaluación, es decir, cómo ponen en acto de dichas reglamentaciones en el día a día escolar. Luego, me detengo en la autocomprensión que hacen de su tarea, a partir de compromisos, rechazos y valoraciones.

\section{Otras prácticas a partir de nuevas demandas}

En los relatos las docentes sobre sus prácticas de evaluación, pude reconocer algunos indicios de la introducción de la gubernamentalidad como forma de ejercicio del poder en el ámbito escolar. Particularmente, tuve en cuenta su referencia a la atención a la diversidad, la intensificación de tareas de registro, de contención psicológica de los niños, de comunicación con padres y a su relación con autoridades escolares; lo cual muestra, entiendo, una ruptura con el modelo del examen propio del ejercicio de poder disciplinario.

\section{La atención a la diversidad}

En sus dichos, las maestras dan cuenta que reconocen la diversidad de sus alumnos, entienden que todos y cada uno de ellos debe aprender; ponen énfasis en el proceso. Asumen dicha tarea desde un compromiso personal. Señala una de ellas:

Nosotras decimos, ¿a este niño pretendemos exigirle? ¡Mirá la vida que lleva! Y uno le tiene que exigir, porque nosotras somos así. No es que 'pobrecito' no podemos hacer más nada por él y lo dejamos. Bueno, pelearé todo el año, es una forma de decir, persiguiéndolo, exigiéndole, llamando a los padres, 'hinchándolo'.

La atención a la diversidad no implica para las maestras entrevistadas aprobar sin que los alumnos tengan los conocimientos necesarios, sino trabajar con quienes tienen dificultades para que los alcancen, como se advierte en el párrafo transcrito.

Por otra parte, se muestran de acuerdo con la problematicidad de la inclusión de niños con dificultades de distinto tipo; hicieron referencia a la complejidad de atender las particularidades de aprendizaje con el número de alumnos que tienen por grado y lo inefectivo de algunas medidas promovidas por el gobierno escolar, por ejemplo, la derivación a centros de apoyo. Además, señalan que hacen todo lo posible, junto con la institución, para que el chico no solo asista a clases y aprenda, sino para que se incorpore a otras actividades como salidas recreativas. La docente de música explica con claridad: "hay algunos que no tienen flauta, que yo les presto y hacen lo que pueden acá. Porque no todos tienen, a veces, en las clases. No se las compran. Entonces, bueno, la escuela me ha comprado algunas".

Para posibilitar la inclusión educativa, introducen diferentes criterios para aprobar las evaluaciones, por ejemplo, la atención a las posibilidades y condiciones reales de los chicos y los niveles alcanzados por la mayoría para no perjudicaros con las notas. Dice la profesora de Educación Física:

\begin{abstract}
Tenés que ver muchas cosas. En mi hora el chico que es, que tiene sobrepeso, que es gordito y le cuesta. Pero, tampoco lo puedo matar con la nota. ¿Qué culpa tiene él de tener ese sobre peso? Y yo lo voy a torturar, si no lo que necesito es que le guste hacer actividad física.
\end{abstract}

Las maestras asumen la ardua tarea de un seguimiento personalizado de los niños, atendiendo las diferencias de aprendizaje y de condiciones. En este sentido, se apropian de la demanda de inclusión educativa como exigencia individual.

\section{Intensificación de tareas: registro y contención psicológica}

Durante las observaciones pude advertir distintas actividades que deben realizar las docentes, por ejemplo, la organización y los ensayos de los distintos grupos para el Festival para el Día de la Tradición, particularmente, las profesoras de Educación Física y Música. Además, el día del Festival estuvieron desde la mañana hasta que terminó el evento en la noche. Muchas veces, la directora estaba a cargo de un curso, porque la docente del mismo estaba ausente; asimismo, comentaba distintas planillas e informes que tenía que enviar a la Dirección General de Escuelas. Las maestras manifestaban las tareas de registro, elaboración de planillas y de planes de recuperación de contenidos que deben completar; lo cual, se suma a la corrección que realizan de pruebas y trabajos. Particularmente, la confección de las Planillas de Seguimiento constituye una demanda que intensifica la tarea docente, ya que supone un registro detallado del desempeño de cada alumno, lo cual conlleva, incluso, a la multiplicación de registros, uno borrador elaborado manualmente y luego, otro confeccionado en computadora.

Por otra parte, reconocen que deben cumplir funciones que exceden el rol tradicional de enseñanza centrado en la transmisión de contenidos, dada la 
realidad de los niños con los que trabajan. Atienden a problemas de índole personal o social. La docente de apoyo, considera que la tarea fundamental del docente se vincula con enseñar a manejar las emociones, ya que los niños tienen reacciones que deben controlar y que dan cuenta de situaciones familiares complejas. Enfatizó: "yo he tenido acá, niños que se sientan y se ponen a llorar. Conmigo, se ponen a llorar". Las docentes señalan que también deben contener en muchos casos los propios padres, una de ellas comentó: "muchas veces, han venido padres, y yo los he sentado, $y$ les he hablado mucho, a final, algunos se han puesto a llorar".

\section{Comunicación con los padres}

Las maestras buscan que el niño apruebe, para ello, entienden que los padres deben involucrarse activamente. Frente a situaciones de bajo rendimiento, contactan a los padres mediante distintas estrategias: pedir que firmen las pruebas, interceptarlos en la puerta de la escuela, llamarlos por teléfono o citarlos mediante una nota que lleva el celador. Una de ellas señala: "porque a los padres nosotras los vivimos llamando y pidiéndoles que hagan que sus hijos estudien". Sin este involucramiento, es muy difícil que se resuelvan las dificultades de aprendizaje de los niños, coinciden en señalar. Sin embargo, ellos no siempre responden "cuesta horrores, cuesta horrores que el padre se comprometa, que lo ayude, es muy difícil", comenta finalmente.

\section{Relaciones con las autoridades: descontento y cautela}

En primer lugar, las docentes refieren a las autoridades educativas como un conjunto indiferenciado, en el que se encuentran directivos, supervisores, capacitadores o autoridades de la DGE. Términos como los de arriba, ellos, señores que están en los escritorios son utilizados para referirse a las autoridades educativas. Advertí que indican situaciones en las que son subordinadas, es decir, los momentos en que las autoridades las ponen en condición de inferioridad. Cuando son capacitadas, interpeladas o se sienten desprestigiadas; particularmente, las docentes se reconocen como último eslabón de una cadena por la que bajan distintas decisiones: "no me sirve que me mande esto, y yo le tenga que dar una devolución. No me sirve. De la manera que lo hacen, no sirve a nadie".

Sin embargo, desde esta posición subordinada, entablan una afirmación de sí mismas, anclada en su saber hacer vinculado a la realización de las tareas cotidianas. Así, las docentes rechazan algunas decisiones del gobierno escolar por considerarlas equivocadas; por ejemplo, las vinculadas al proceso de enseñanza aprendizaje, a la evaluación o a la promoción de los chicos. Entienden que las capacitaciones a las que asisten están equivocadas en sus propuestas y argumentan desde su saber hacer para demostrarlo. Por ejemplo, una docente de Matemática y Ciencias Naturales se quejó de los distintos programas de capacitación cuyas propuestas deben seguir con posterioridad en sus clases. También, rechazaba la valoración de las autoridades sobre la repitencia de los alumnos:

Ellos hablan mucho, que los traumamos, que esto, lo otro. No sería mejor, por ejemplo, no sé porque tienen tanto problema con que el niño haga sala de 5 dos veces, que sería lo ideal. Si el niño no está preparado... No sé porque tienen tanto problema con eso, hacer dos veces un año.

Desde su experiencia, las maestras argumentan que repetir mejora los aprendizajes. Siempre utilizan el impersonal, ellos, te dicen, te bajan para referirse a quienes les indican lo que tienen que hacer y no están como ellas frente al aula. Evidenciado que son puestas en situación de inferioridad, ya que deben hacer lo que les dicen.

Una queja frecuente es la referida a las jornadas ${ }^{8}$ que habitualmente se hacen en la escuela, las rechazan por considerarlas inefectivas para la resolución del día a día escolar. “Es que en las Jornadas nos llenan de contenido teórico, denso, aburrido que a nadie le interesa, que no aporta nada, ... en definitiva no nos dan espacio para que nos juntemos", comenta una docente. Asisten, pero, les parecen poco significativas; el argumento se refiere a su alejamiento de la práctica, que es lo que ellas quieren resolver. Desde esta valoración de la práctica y de su saber hacer, aprecian las instancias de diálogo que se abren desde la supervisión como los ateneos. ${ }^{9}$ En estos encuentros ellas pueden compartir sus experiencias y centrarse en resolver con sus pares de área modos de hacer, de enseñar; aprecian el poder trabajar juntas: "si, eso, un ateneo de lengua realmente te sirve. Nos sirvió porque está orientado a la práctica. Nosotros necesitamos cosas orientadas a la práctica".

8 Las Jornadas son reuniones que la DGE indica para las escuelas con contenidos definidos. Se desarrollan a lo largo de una mañana generalmente. También a comienzos de año existes estos momentos para trabajar sobre los lineamientos propuestos por las autoridades escolares. Para estos encuentros la DGE elabora un material que es trabajado en las escuelas con la guía de los directivos.

9 Los Ateneos, son reuniones de docentes de distintas escuelas, por área del conocimiento que propone la DGE, generalmente asociada a los cambios a nivel curricular. Se desarrollan a nivel de sección escolar. 
Pese al descontento, no hay una oposición organizada para romper con las dinámicas planteadas. Las docentes se fastidian por lo que dicen en términos generales las autoridades, pero no proponen, por ejemplo, negarse a las jornadas o reuniones de personal. Tampoco, las diferencias con las autoridades implican un conflicto que tensione las actividades escolares. Ellas no se rebelan frente a lo que consideran incorrecto, solo se enfadan. No enfrentan a las autoridades, hacen lo que les piden, aunque no estén de acuerdo.

En segundo lugar, reconocen su situación de vulnerabilidad frente a la interpelación de las autoridades educativas. Las docentes, según establece la reglamentación, tienen que llevar una Planilla de Seguimiento, con los indicadores para reconocer los logros alcanzados por los niños, que se completa con números. El párrafo siguiente muestra su relevancia y la presión que ejerce el juicio de las autoridades:

porque siempre uno tiene eso que, si superan el 25 \% los desaprobados, bueno, el cuestionado es el docente. Porque, lamentablemente es así. A fin del trimestre, cuando se llena la planillita del 25\%, te empiezan a preguntar, ¿qué pasó acá? Este año no me paso, pero el año pasado sí. Tenía muchos desaprobados en Ciencia Sociales. ¿Y por qué tantos? En Lengua no tantos ¿por qué? [...] Uno siempre tiene en cuenta eso.

Si bien, la interpelación aparece en espacios informales, desde la perspectiva docente se vive como una demanda que pone en duda su propio su propio desempeño, el procedimiento se da en la institución, pero también desde la supervisión. Refiriéndose a la asignación de nota (calificación numérica), una de las docentes deja en claro, presiones que reciben de directivos y supervisores para ajustarla en función de la permanencia de los niños en la escuela. Señala: "no te dicen no desaprobés. Pero, a ver chicas fíjense. Por eso te digo, nosotras somos muy cautelosas después de muchos años, ya creo que hemos aprendido a manejar esa situación también".

Así, las docentes dan cuenta de los cuestionamientos y presiones que reciben de parte de las autoridades para que revisen su propia actuación, a partir de las notas en la Planilla de Seguimiento. La responsabilidad por los resultados es del docente, que debe lograr que los niños aprendan y responder por ello cuando no lo han hecho, según las notas que presentan. Ellas perciben en esta situación cierta intimidación. De ahí, que se definan como cautelosas. La autorreflexión de las docentes sirve como instancia de direccionamiento de las mismas por parte de las autoridades. Por ello, las docentes entienden que deben evitan problemas, así, revisan las notas y las planillas respondiendo a las demandas de las autoridades.

\section{Reivindicar el "guardapolvo blanco": Saber hacer, reconocimiento y autovaloración}

En las entrevistas también pude advertir la autoafirmación que de sí mismas realizan las docentes. Todas argumentaban claramente sobre sus posicionamientos desde la propia experiencia, ponderando lo positivo y negativo que las había llevado a modificar rutinas, a generar propuestas personales y a contemplar diferentes factores en la evaluación. Esta autoafirmación, entendida como profesionalidad, se sustenta en su propia tarea cotidiana, en la cual han desarrollado un saber hacer surgido de la reflexión sobre su propia experiencia y de su ética personal. Plantean, así, una diferencia con colegas y otros profesionales. Por otra parte, en sus dichos ponderan el reconocimiento que de su tarea hacen las familias de la comunidad.

\section{La profesionalización: saber hacer, responsabilidad y autonomía}

Esta reflexión sobre su desempeño se vincula, en primer lugar, un saber hacer resultado de su experiencia y de una autocrítica de sus decisiones frente a situaciones complejas. Este saber hacer es un conocimiento de lo particular, pero vinculado a criterios y posturas que exceden lo inmediato para constituirse en criterios seguros, basados en su experiencia. Como señala una de las docentes de Lengua y Ciencias Sociales: "en realidad, fui descubriendo por iniciativa propia, porque no es algo que yo lo traía de mi formación docente...Porque tenés que hacer autocrítica, porque tenés que decir, qué hice mal antes para no lograr tal cosa". Este saber hacer tanto en la evaluación como en las demás tareas que realizan se combina con la rigurosidad y la autonomía que definen su carácter profesional. Es significativo el uso del lenguaje ético con el que ellas se refieren a su práctica. Ponen énfasis en la responsabilidad que tienen, que no depende de la vigilancia de otros, sino de sí mismas y de su compromiso con la educación de los niños. Advierten diferencias con sus colegas, algunas no cumplen con lo establecido, incluso enseñan con errores. Las entrevistadas marcan la responsabilidad individual y asumen su tarea con un fuerte compromiso moral:

Cada docente tiene que ser responsable, en el sentido, de qué enseño y cómo lo enseño. Y eso es algo, que a mí me parece que va por otro lado, no en la institución, en el control institucional, estar ahí, en uno.

Como se puede reconocer en los dichos de las docentes, hay un compromiso moral que consideran necesario asumir como profesionales frente a las demandas que reciben diariamente, fundamentalmente 
vinculadas al aprendizaje de los niños. Su compromiso es ético porque no debe depender del control sino de la iniciativa personal, incluso frente a las dificultades. Compromiso con el acompañamiento de cada niño, con la dedicación para que alcancen los logros esperados. Una de ellas afirma con contundencia: "uno siempre lo sigue intentando. La esperanza es lo último que se pierde, no podría trabajar como maestra si pensara que no tengo nada que hacer por los chicos".

\section{El reconocimiento de la comunidad}

Las docentes encuentran su fortaleza en el reconocimiento de la comunidad. Advierten que sus exigencias o su forma de actuar constante a lo largo de los años han consolidado una imagen positiva, la cual es la base de su relación con los padres. Para ellas, lo importante es que las familias de los niños valoran sus acciones. Una de las docentes de Lengua y Ciencias Sociales comentó: “¿cuál es la ventaja nuestra? que los padres nos conocen, la comunidad nos conoce, hace muchos años que trabajamos acá, saben cómo somos". El aprecio de las familias por su trabajo y por las exigencias que proponen para el aprendizaje es la mejor recompensa que tienen las maestras, es signo de su profesionalidad.

Cuando terminé de grabar una de las entrevistas, la docente comentó que las estudiantes de profesorado que hicieron sus observaciones en la escuela habían ido mal vestidas. Fue entonces, cuando reivindicó el uso del guardapolvo blanco, ${ }^{10}$ como símbolo de su profesionalidad. Explicó que las docentes de la escuela habían acordado usar esa prenda, porque los otros [guardapolvos de colores] los usan también en la verdulería. Hizo referencia a la profesionalidad, a la necesidad de que valoren su tarea de enseñar. Afirmó que el esfuerzo y la dedicación son signos de la responsabilidad que asumen por decisión propia.

\section{Conclusiones: ¿una nueva forma de ser maestra?}

Como señalé en la introducción, me interesa centrar la reflexión sobre el ser maestro a partir de una perspectiva que posibilite contextualizar su tarea y repolitizar su desempeño como agente público. Pensar políticamente no significa considerar lo que ocurre en las escuelas como efecto de las decisiones de los gobiernos

10 En Argentina, a partir de la década de 1940 fue obligatorio para los docentes el uso de un guardapolvo blanco. Con posterioridad a 1983, es indistinto el color y el diseño de los mismos. Sin embargo, el guardapolvo blanco en nuestro país es considerado un símbolo de la educación pública y del valor de los docentes. justificadas desde distintas posiciones ideológicas, sino dar cuenta de la orientación de las medidas educativas que mantienen un mismo direccionamiento pese a los cambios en la gestión. La perspectiva de los estudios de gubernamentalidad permite evidenciar la complejidad de la acción docente, particularmente, en instituciones a las que asisten niños y niñas de sectores populares. Porque muestra la trama de relaciones de poder en la que se lleva adelante y permite visualizar el modo en como el Estado busca dirigir la conducta de la población a través de la educación, desde políticas que suponen la gubernamentalidad neoliberal como modo de ejercicio del poder.

Particularmente, me pregunté por el impacto de las nuevas reglamentaciones sobre la evaluación de los aprendizajes en las prácticas docentes y la autocomprensión que tienen los docentes de sí mismos, a partir de la Ley de Educación Nacional № 26.206, cuyo objetivo es la inclusión educativa en un marco de desigualdad y exclusión social. Partí del supuesto de que las reformas, que apuntan a reemplazar la evaluación tradicional por una formativa, rompen con el modelo del examen propio del disciplinamiento e introducen el monitoreo afín al ejercicio de la gubernamentalidad. La metodología etnográfica ${ }^{11}$ me permitió identificar en los relatos de docentes de una escuela de sectores populares cómo ponen en práctica las reformas y cómo esto modifica su modo de reconocerse como maestras.

En los dichos de las docentes advertí las modificaciones en las prácticas de la evaluación que llevan adelante a partir de la incorporación de pautas de las nuevas reglamentaciones. Allí se puede reconocer, entiendo, indicios de la ruptura del modelo del examen tradicional, propio del disciplinamiento, y la introducción del monitoreo como forma de evaluación (1). Estos cambios inciden en la comprensión que las maestras entrevistadas tienen de su tarea, y de sí mismas. Asimismo, muestran el modo en que ellas mismas son conducidas por las autoridades educativas, y también, se evidencia la forma en la que ellas rechazan sus decisiones y se autoafirman como profesionales (2).

1. Las maestras, según la interpretación que hacen de sus prácticas, no controlan simplemente los aprendizajes de sus alumnos, deben acompañarlos, incentivarlos, lograr que pese a sus dificultades aprendan y convencerlos de que pueden hacerlo. Por otra parte, entienden que deben comunicarse con los

11 Los estudios etnográficos de un caso único no conllevan a una generalización de sus conclusiones. Su potencial criticó radica en que muestran desde un registro denso las relaciones entre los distintos sujetos junto con los sentidos atribuidos por ellos a su acción (Marradi, Archetti y Piovano, 2010). 
padres para que intervengan en el aprendizaje de sus hijos. Sus prácticas están más cerca del monitoreo y del modo de ejercicio propio de la gubernamentali$\mathrm{dad}$, en cuanto asumen funciones de seguimiento, motivación y atención a las diferencias. No mandan, ni obligan; conducen a los niños, persuadiéndolos a ellos y a sus familias de que pueden lograr las metas propuestas, interviniendo calculadamente. Estimulan que cada uno acreciente sus propias posibilidades, siempre se puede lograr más.

El docente en el modelo escolar tradicional debía tener un manejo de los conocimientos para enseñarlos de la mejor forma posible, debía controlar que todos los aprendieran e identificar a quien no lo hacía o pretendía engañarlo, siguiendo el modelo del examen. Debía vigilar para que todos hicieran la misma tarea, comparar, calificar y jerarquizar al interior del grupo clase para que los niños bajo el incentivo de la competencia buscaran aprender más y mostrar su excelencia. El acercamiento de docente a algún niño o familia, en particular, era calificado como parcialidad o favorecimiento. El docente debía permanecer distante de los niños para no mezclar afectos con evaluación de conocimientos. Debía ser un buen árbitro, manteniendo la distancia que le garantizaría imparcialidad a la hora de la evaluación.

Según los relatos de las entrevistadas, su relación con niños y niñas ha cambiado, implica un mayor conocimiento y desarrollo de su dimensión afectiva para llevar adelante las prácticas escolares cotidianas. Las intervenciones de las docentes son calculadas para que cada uno de los chicos y chicas alcancen los logros esperados a partir de sus propias posibilidades. No hay sanción, sino acompañamiento y reconocimiento de posibilidades; no hay vigilancia sino comunicación y estimulación. La relación con los padres también se ha modificado, no solo les comunican los resultados, sino que los conminan a involucrarse con el aprendizaje de sus hijos. Lejos de ser un árbitro, las docentes responden al modelo del entrenador, quien sigue individualmente y exige de acuerdo a las propias posibilidades de cada niño, comprometiendo a los adultos a intervenir para que efectivamente alcance los logros esperados

2. Desde los relatos de las maestras se evidencian no solo cambios en las prácticas de evaluación, sino que pueden advertir indicios de una incipiente reconfiguración de su subjetividad como docentes:

a. Asumen como propia la demanda de atención a la diversidad: las docentes entrevistadas comentan experiencias concretas que evidencian como parte de su práctica la promoción de la igualdad de los niños en la vida escolar. También, realizan acciones de acompañamiento, estímulo, contención afectiva de los niños. Se involucran afectivamente con cada uno para facilitar su aprendizaje. La contención afectiva e incluso la educación de las emociones de los niños, particularmente de quienes tienen dificultades, es parte de su tarea cotidiana. Además, entablan una relación de seguimiento y contención con los padres.

b. Desarrollo de capacidades psicológicas y de comunicación: la atención a la individualidad de los chicos y chicas suscita un desarrollo de sus cualidades psicológicas para considerar la dimensión afectiva de sus alumnos, además de sus posibilidades de aprendizaje. Lo cual implica, evidentemente, una psicologización de la tarea docente, en la medida que se apropian de la estimulación y en el seguimiento personal de los alumnos, no solo de desarrollar contenidos de distintas asignaturas (modelo docente tradicional). Además, la necesidad de implicar a los padres potencia en los docentes las cualidades de comunicación. Para involucrarlos en el aprendizaje de sus hijos deben escucharlos, orientarlos y comprometerlos. Para ello, las maestras deben ser creativas, persuasivas y desarrollar su capacidad de comunicación.

c. Situación de subordinación y vulnerabilidad: las docentes consultadas perciben su situación de sujeción frente a las autoridades que les dicen lo que tienen que hacer, la forma en la que tienen que enseñar y los métodos a utilizar. Evidencian y reconocen su situación de subordinación, porque siempre son capacitadas por otros, reciben indicaciones que les bajan, asisten a jornadas en las que se justifican las medidas de gobierno, pero, rara vez, deciden ellas mismas. Además, advierten su situación de vulnerabilidad porque perciben el cuestionamiento de su desempeño al ser interpeladas por directivos y supervisora por los niños que aparecen desaprobados en las planillas. Por otra parte, bajo el imperativo de la efectividad y responsabilizadas como agentes primarios del rendimiento de sus alumnos, ellas mismas son acompañadas, guiadas y exigidas desde los resultados. Lo que evidencia que son conducidas también de forma gubernamental. Son conducidas, específicamente, a partir de la autoevaluación que deben realizar revisando la Planilla de Seguimiento. Las autoridades las conducen para que revisen sus prácticas de modo que sean cada vez más efectivas, a fin de garantizar el aprendizaje de todos y cada uno de los niños. 
d. Saberesy prácticas de autoafirmación: las docentes entrevistadas han modificado sus prácticas, atienden a las diferencias, desarrollan capacidades psicológicas y comunicativas, acompañan y alientan. Lo cual genera como contrapartida una afirmación de sí mismas como profesionales. Interpretan sus acciones poniendo en valor su saber hacer docente, es decir, aquellos conocimientos aprendidos en el día a día escolar que les permite resolver dificultades. Ellas han aprendido a conocer los niños, a cómo deben enseñar distintos temas, cuánto y cómo deben exigir y cuándo reconocer las limitaciones personales. Desde su saber hacer guían los aprendizajes individualmente, y también, rechazan específicamente decisiones del gobierno. Su experiencia es valiosa, pese a no ser reconocida por quienes les dan teorías o están en los escritorios. La apreciación que las familias hacen de su labor es el reconocimiento social indiscutible de su trabajo, su orgullo. La comunidad, señalan las maestras, sabe cómo somos. Como sintetiza la directora: esta es una escuela elegida.

En síntesis, las maestras entrevistadas acompañan e incentivan a los niños en sus aprendizajes, atienden diferencias y buscan alentar y optimizar sus aprendizajes. Sus prácticas, cercanas a las de un entrenador, remiten a la persuasión y no al miedo, además, potencian las acciones de cada alumno desde una intervención calculada. Ellas orientan, detectan riesgos, suscitan la autoevaluación y promueven una conducta llamada a revisarse, a aprender y mejorar de modo permanente. Lo cual muestra indicios de una nueva configuración docente vinculada particularmente a conocimientos psicológicos y rasgos de personalidad, ya que deber dirigir particularmente a cada alumno (Bernad, et ál., 2015). Las docentes entrevistadas desarrollan capacidades psicológicas, habilidades de comunicación que les permite valorar su saber hacer ligado a la resolución de situaciones cotidianas. Así, se subjetivan como agentes activos de la educación, asumen un protagonismo basado en un saber hacer fruto de su experiencia y reconocen como desafío el aprendizaje de todos sus alumnos. Se definen como profesionales y rechazan decisiones de gobierno. Evidenciado una incipiente contraconducta cercanas a la mística, ${ }^{12}$ desde su definición como profesionales, simbolizada con el guardapolvo blanco (Foucault, 2006, p. 256).

12 M. Foucault entiende por contraconducta, una lucha contra los procedimientos puestos en práctica para conducir a los otros. El uso del término contraconducta pone de relieve no a las personas, sino a la manera concreta de actuar de alguien en un campo de relaciones de poder (Foucault, 2006, p. 239). El filósofo francés considera que, en la Edad Media, se advierten
Entiendo que, desde el sentido que ellas atribuyen a sus acciones, no pueden comprenderse como una resistencia pasiva, en la medida en que no son un tope al direccionamiento de la conducta que ejercen las autoridades. Ellas no son reacias a cumplir con las obligaciones que les imponen (Foucault, 2006, p. 224). Por el contrario, asumen las demandas de atención a la diversidad, de nuevos vínculos con los estudiantes y familias, y realizan las tareas de registro. Rechazan, específicamente, el modo de ser conducidas por las autoridades escolares, porque no valoran sus saberes. Las docentes entrevistadas dan cuenta de una experiencia que se desarrolla y autoafirma con independencia de quienes las conducen, lo hacen en términos morales de responsabilidad y conciencia profesional.

El uso de categorías propias de los estudios de gubernamentalidad para analizar la evaluación escolar a partir de las reformas implementadas desde la LEN № 26.206 muestra, por un lado, la insuficiencia de pensar las prácticas docentes desde el disciplinamiento; y por otro, a través de estas herramientas teóricas podemos comprender la relación entre las decisiones políticas y lo que ocurre con las prácticas docentes en el día a día escolar. En esta investigación, particularmente, pude identificar la direccionalidad de las políticas educativas que introducen una forma de conducción de la conducta, el monitoreo, a través de la modificación de la evaluación de los aprendizajes escolares. Asimismo, comprender cómo las docentes entrevistadas reconstruyen activamente el sentido de su tarea cotidiana y la autocomprensión que tienen de sí mismas. Se reconocen como profesionales, mostrando, una nueva forma de ser maestra.

Al ser interrogadas por la evaluación de los aprendizajes ninguna de las docentes utilizó el término justicia. Por ello, entiendo que las modificaciones de las prácticas docentes nos invitarían a pensar sobre las nuevas formas de gobernar del Estado. El Estado argentino, particularmente, prometió justicia a través

cinco formas de contraconducta que tienden a redistribuir, invertir, anular o descalificar el poder pastoral: el ascetismo, las comunidades, la mística, el problema de la Escritura y la creencia escatológica. La mística consiste en una experiencia individual secreta en la que el individuo se comunica con Dios, su llamado le exige una respuesta personal de fidelidad. Este vínculo permite a cada uno ser su propio juez, evaluándose desde su propia conciencia. Lo cual genera una distancia frente a quien pretende conducirlo, el sacerdote (Foucault, 2006). La mística posibilita la ruptura con el direccionamiento que ejercen los pastores, sin recusarlos. Por otra parte, define la resistencia pasiva como topes externos que implican incumplimientos de las obligaciones impuestas por los pastores (Foucault, 2006). 
del Sistema Educativo durante el siglo pasado (Tenti, 2004). Por medio de la escuela obligatoria configuró ciudadanos que creían en la justicia generada a través de los exámenes. El docente estricto ejercía su autoridad en la evaluación, lo cual proyectaba un modelo de Estado que asumía una función de juez imparcial frente a los ciudadanos.

Hoy, también, el Sistema Educativo es una manera particular de gobernar a la población a través de la escolarización de niños y jóvenes. No la única, por supuesto, pero, desde lo aquí presentado, me animo a conjeturar que el Estado argentino, en el presente siglo, establecería otra relación con los individuos, atendiendo a otro modo de conducir su conducta, desplegado en la escuela, a través del establecimiento de relaciones de poder gubernamental, introducidas por un tipo de evaluación a fin al monitoreo. Un docente más entrenador y menos juez, con más conocimientos de psicología y el desarrollo de la comunicación. Un maestro que debe dar cuenta de su actuación a partir de los logros alcanzados por sus alumnos.

Desde la escuela, modificando el modo de evaluación, el Estado convalidaría, entiendo, su propia modalidad gubernamental de actuación mínima, calculada, que deja en libertad, pero también en soledad a cada uno de los ciudadanos. La nueva manera de ser docente, primer agente público con quien se encuentra un niño, proyectaría otra forma de comprender la vinculación el Estado con los ciudadanos. Un Estado entrenador y no un árbitro. Frente al Estado no cabrían los reclamos de justicia, porque acepta que el juego, es decir, la dinámica social no depende de él, porque se limita a dar herramientas para que cada uno resuelva sus problemas como pueda. Sin embargo, los indicios de contraconducta de las docentes nos invitarían a seguir indagando sobre las posibilidades de revertir esta situación en la misma escuela. En la medida que la autoafirmación de sí mismas que hacen las maestras, articulada en una mística su compromiso con el aprendizaje de los niños, supondría una revalorización de la tarea educativa con independencia de los direccionamientos gubernamentales.

\section{Referencias}

Ball, S. (2001). La gestión como tecnología moral. Un análisis ludista. En S. Ball, Foucaulty la educación. Disciplinas y saber [Cuarta Edición] (pp. 155-167). Morata.

Beech, J. y Meo, A. (2016). Exporando el uso de las herramientas teóricas de Stephen Ball en el estudio de las políticas educativas en América Latina. Archivos Analíticos de Politicas Educativas, 24(23), 1-19.
Benza, G. (2016). La estructura de clases argentina durante la década 2003-2013. En G. Kessler, La sociedad argentina hoy. Radigrafía de una nueva estructura (pp. 112139). Siglo XXI Editores, Fundación OSDE.

Bernad, I. y García, J. (2015). La trasformación de las politicas educativas desde la gobernamentalidad neoliberal: el caso español como ejemplo. En N. Dal Ri, T. Suely Antonelli y M. Bravo (Eds.). Políticas educacionais, gestão democrática e movimentos sociais: Argentina, Brasil, Espanha e Portugal (pp. 149-172). Cultura Acadêmica Editora.

Foucault, M. (1989). Vigilar y castigar, el nacimiento de la prisión. Siglo XXI.

Foucault, M. (2001). Post-scriptum. El sujeto y el poder. En H. Dreyfys y P. Rabinow (Eds.). Michel Foucault: más alla del estructuralismo y la hermenéutica (pp. 241-259). Ediciones Nueva Visión.

Foucault, M. (2006). Seguridad, territorio y población. Fondo de Cultura Económica.

Foucault, M. (2007). El nacimiento de la biolpolítica. Fondo de Cultura Económica.

Gluz, N. (2016). Políticas y prácticas en torno a la 'inclusión escolar ' ¿Por qué es tan dificil la democratización escolar? Estación Mandioca de Ediciones S.A.

Grinberg, S. (2008). Educación y poder en el siglo XXI. Gubernamentalidad y pedagogía en las sociedades del gerenciamiento. Miño y Dávila.

Grinberg, S. (2015). De la disciplina al gerenciamiento, del examen al monitoreo. Un estudio sobre el gobierno y la evaluacón en las sociedades contemporáneas. RASE Revista de la Asociación de Sociologia de la Educación, $8(2), 155-172$. http://rase.ase.es

Ley de Educación Nacional N²6.206 en https://www. argentina.gob.ar/sites/default/files/ley-de-educ-nac58ac89392ea4c.pdf

Marradi, A., Archenti, N. y Piovano, J. I, (2010). Estudio de caso/s. En Metodología de la investigación en Ciencias Sociales.. CENGAJE Learning.

Miranda, E. (2011). Una 'caja de herramientas' para el análisis de la trayectoria de al politica educativa. La perspectiva de los ciclos de la politica. En E. Miranda y N. Paciulli (Eds.). (Re) Pensar la Educación Pública. Aportes desde la Argentina y Brasil (pp. 105- 126). Editorial de la FFYL UNCórdoba.

Noguera, C. y Marín, D. (2012). Educar es gobernar: la educación como arte de gobierno. Cadernos de Pesquisa, 42(145), 14-29.

Noguera, C. y Marín, D. (2013). Biopolítica y educación: hacia una nueva crítica de la educación. Entrevista a Silvia Grinberg. Pedagogía y saberes, (38), 115-124. 
Rose, N. (1990). El gobierno del alma. La formación del yo (self) privado. www.elseminario.com.ar/biblioteca/ Rose_gobierno_alma_cap1_4.htm.

Rose, N. (2007). ¿La muerte de los social? Reconfiguración del territorio de gobierno. Revista Argentina de Sociologia, 5(8), 111-150.

Semán, P. y Ferraudi, C. (2016). Los sectores populares. En G. Kessler (Ed.). La sociedad argentina hoy. Radiografia de una nueva estructura (pp. 141-162). Siglo Veintiuno Editores.

Tenti, E. (2004). Sociología de la Educación. Universidad Nacional de Quilmes Editora.

Tosoni, M. (2019). Otro modo de evaluar: la intoducción de relaciones de gubernamentalidad a través de la evaluación de los aprendizajes. Análisis de caso. III Congreso Latinoamericano de Teoria Social: Desafíos contemporáneos de la Teoria Social Mesa 20. http:// diferencias.com.ar/congreso/ICLTS2015/wp/index. php/mesas-tematicas-3/.

Varela, J. (2001). Prólogo a la edición española. En S. Ball, Foucault y la educación. Disicplinas y saber. 4aㅡ Edición, (pp. IX - XV). Ediciones Morata.
Veiga-Neto, A. y Saravia, K. (2011). Educar como arte de gobernar. Curriculum sem fronteiras, 11(1), 5-13. http://www.curriculosemfronteiras.org/vol11iss1articles/veiga-neto-saraiva.pdf

Veiga-Neto, A. (2013). Biopolítica, normalización y educación. Cuadernos de pensamiento biopolítico latinoamericano No 1 Actas del III Coloquio Latinoamericano de Biopolítica y I Coloquio Internacional de Biopolítica y Educación (10-19). Buenos Aires: Unipe Editorial Universitaria. https://editorial.unipe.edu.ar/cuadernos/cuadernos-de-pensamiento-biopolitico-latinoamericano

Verger, A. y Normand, R. (2015). Nueva gestión pública y educación: elementos teóricos y conceptuales para el estudio de un modelo de reforma educativa global. Educação \& Sociedade, 36(132), 599-622.https://doi. org/10.1590/ES0101-73302015152799

Yuni, J. y Urbano, C. (2005). Mapas y Herramientas para conocer la escuela. Investigación Etnográfica e Investigación Acción. Brujas. 